Cahiers de recherches médiévales

\title{
Défense et illustration de la guerre
}

Le Jouvencel de Jean de Bueil

\section{Marie-Thérèse de Medeiros}

\section{(2) OpenEdition}

Journals

Édition électronique

URL : https://journals.openedition.org/crm/1452

DOI : $10.4000 / \mathrm{crm} .1452$

ISSN : 1955-2424

Éditeur

Honoré Champion

\section{Édition imprimée}

Date de publication : 30 octobre 1998

Pagination : 139-152

ISSN : $1272-9752$

Référence électronique

Marie-Thérèse de Medeiros, «Défense et illustration de la guerre », Cahiers de recherches médiévales [En ligne], 5 | 1998, mis en ligne le 30 novembre 2009, consulté le 15 décembre 2022. URL : http:// journals.openedition.org/crm/1452; DOI : https://doi.org/10.4000/crm.1452

Ce document a été généré automatiquement le 15 décembre 2022.

Tous droits réservés 


\title{
Défense et illustration de la guerre
}

\author{
Le Jouvencel de Jean de Bueil
}

\author{
Marie-Thérèse de Medeiros
}

1 Alléché par un titre prometteur, attiré par la présentation que fait Aragon de cet ouvrage qu'il range aux côtés du Petit Jehan de Saintré parmi les premières manifestations du roman réaliste ${ }^{1}$, un insuffisant lecteur- $s^{\prime}$ il en est!- pourrait se trouver fort déçu à la lecture du Jouvencel, que son auteur qualifiait de «petit traictié narratif $»^{2}$. L'intrigue est en effet des plus banales dans ses grandes lignes - l'irrésistible ascension d'un pauvre et jeune noble au sommet de la hiérarchie sociale par la carrière des armes- et l'essentiel du récit tourne autour de la guerre, aussi bien dans ses aspects pratiques et tactiques, que dans ses rapports avec le droit et l'idéologie. C'est du reste en tant que traité de guerre que l'ouvrage de Jean de Bueil figure en compagnie de l'Arbre des batailles, composé trois quarts de siècle auparavant par le bénédictin Honoré Bouvet et, entre autres, du Livre des faits d'armes et de chevalerie de Christine de Pizan, rédigé voire "compilé» en 1409-1410 ${ }^{3}$, dans le chapitre du G.R.L.M.A. où Philippe Contamine analyse «les Traités de Guerre, de Chasse, de Blason et de Chevalerie» qui ont vu le jour aux $\mathrm{XIV}^{\mathrm{e}}$ et $\mathrm{XV}^{\mathrm{e}}$ siècles. Entrelaçant une composante fictionnelle et une composante didactique, Le Jouvencel s'est enrichi, et ce dès sa diffusion, d'une autre dimension, autobiographique, ou plus exactement crypto-autobiographique, en raison $\mathrm{du}$ commentaire qui figure dans quelques manuscrits aux côtés du «petit traitié narratif» de Jean de Bueil et qui est l'œuvre de Guillaume de Tringant. En effet cet écuyer du seigneur de Bueil a jugé bon d'éclairer le lecteur et de lui montrer que faits et personnages «déguisés» sous des appellations de fantaisie ou codées renvoyaient à des événements et personnes historiques gravitant autour de la vie de son maître, qui tient bien entendu le premier rôle, celui du Jouvencel. Fiction romanesque- bien que le nom de roman ne soit pas utilisé par l'auteur-, traité illustré par l'exemple relatif à la guerre, biographie distanciée, c'est sur ces différents aspects de ce récit en prose que je souhaiterais m'arrêter, en examinant les rapports qu'ils entretiennent entre eux.

2 L'ouvrage de Jean de Bueil se présente bien d'abord comme un récit de type romanesque et relate sur de longues années la carrière par les armes «d'un povre jeune gentilhomme $»^{4}$ que l'auteur rencontra au cours d'un voyage qu'il dut faire dans une 
région désolée et ravagée par la guerre. Peu soucieux de faire languir son lecteur sur le sort que l'avenir réserve à ce jeune homme, le narrateur donne d'emblée la source de son récit, les conversations qu'il a eues avec «ce jeune gentilhomme»- «si parlay à lui par plusieurs fois... très doulcement me respondoit, en me racomptant de ses aventures ${ }^{5}$ - et l'aboutissement de son parcours, car après avoir souligné la foi en Dieu de celui que l'on va surnommer peu après le Jouvencel, il ajoute:

Par quoy Dieu lui aida tellement que puis le vey-je prince et seigneur, et posseder regions et terres en sa main, par le grant sens et vaillance et diligence de sa personne $e^{6}$.

3 Ce n'est certes pas dans l'originalité ou l'imprévisibilité de l'intrigue, on le voit, que se situe la force du récit. Il reprend un schéma bien connu, celui des «romans d'un jeune homme pauvre» ${ }^{7}$, dont dès le XIII ${ }^{e}$ siècle le roman en vers de Philippe de Rémi Jehan et Blonde proposait une mise en œuvre très réussie. La construction ascendante, mimant l'ascension sociale du personnage, le conduit de petits coups de main dérisoires aux grands affrontements et aux plus grandes responsabilités militaires et politiques. Et l'auteur n'hésite pas à recourir à une ficelle narrative passablement usée, le mariage avec une fille de roi, pour justifier l'extraordinaire trajectoire du Jouvencel que sa "prouesse», sa droiture et son inébranlable foi en Dieu conduisent au sommet de la hiérarchie sociale. Guillaume Tringant d'ailleurs souligne bien, dans la perspective qui lui est propre, la faiblesse de cet épisode qui pourrait démentir ses révélations sur Le Jouvencel, biographie déguisée selon lui de Jean de Bueil:

vray est qu'il y a une fiction faicte de la fille du roy Amydas et du Jouvencel; pour ce les susdis serviteurs ne voloient pas ne ne veoient le Jouvencel estre tirant ne parvenu à estre seigneur et prince par tyrannie, pour ce que leur livre est faict et fondé sur bonne equité; et autrement n'y povoit parvenir, car il n'avoit nulle cause de soy faire seigneur ne prince, pour ce que c'estoit ung povre gentilhomme; et est fait ce mariage pour exemple de bien faire et pour monstrer que nul ne doit venir à hault estat ny à grant seignorie, s'il n'y vient justement et à bonne querelle... ${ }^{8}$

Guillaume Tringant aurait pu mentionner un autre emprunt manifeste aux œuvres de fiction: la mention de la «damoiselle de Grantfort» ${ }^{9}$ que le roi Amydas qualifie de «plus forte enchanteresse et... plus mauvaise femme du monde $»^{10}$. On peut penser que s'il ne s'y arrête pas c'est d'abord en raison d'une intervention très ponctuelle: elle n'apparaît que dans le dialogue entre le Régent (ci-devant Jouvencel) et son beau-père le roi Amydas, lors d'une séance de conseil tenue dans la ville de Bort. Il est possible aussi que l'écuyer de Jean de Bueil n'ait pas éprouvé le besoin de justifier un développement du récit qui s'inscrit d'emblée dans une rationalisation des pouvoirs magiques liés à l'enchantement. En effet si, dans un premier temps, la présentation que fait le roi Amydas de la dite demoiselle semble l'identifier à quelque Morgane ou Camille- «elle tient une dame en ce pays... et beaucoup d'autres gens de bien", «et a encore davantaige le chastel de Grantfort... là où elle tient encores plus de dix chevaliers et plus de vingt jouvenceaulx en son servaige» ${ }^{11}$, le roi dissipe par la suite toute aura mystérieuse et référence à des forces occultes. En fait le souverain n'attribue les pouvoirs de la demoiselle pour finir qu'à « la force de son chastel et de son beau langaige et de la subtilité et mauvaistié dont elle est pleine» et il ajoute:

Je n'entens point que par son enchanterie elle sceust rien faire; mais elle seduiroit tout le monde par son engin ${ }^{12}$ 
Dépouillée d'un savoir magique qui la distinguerait du commun des mortels, la demoiselle de Grantfort rejoint l'humaine condition et n'est plus qu'une manipulatrice, dotée d'un don de la parole et d'une intelligence qu'elle met au service du mal.

6 Ainsi ce bref emprunt à l'imaginaire romanesque pourrait bien n'avoir qu'une fonction de démystification, renforcée par les protestations du Régent qui souligne que celui qui se fie en Dieu se soucie peu des enchantements, ce qui donne l'occasion à l'auteur de prêter un bon mot à son personnage:

Monseigneur laissez la chanter et enchanter; car je ne la crains riens et n'ay point paour que telles enchanteries me facent ou sceussent faire mal ne desplaisir; car j'ay bonne creance en Dieu. ${ }^{13}$

$7 \mathrm{Si}$, quittant les charmes de la demoiselle de Grantfort, on en revient à la séduction que le texte exerce sur le lecteur dans sa dimension fictionnelle, on goûtera peut-être les fort longs et beaux discours que Jean de Bueil prête à certains de ses personnages, notamment ceux que prononcent trois hommes du grand conseil du roi: Jehan Bienassis, le sire de Chamblay et maître Nycolle. On peut toutefois ne pas apprécier outre mesure cette rhétorique bien huilée, érigée par Paulin Paris au rang de «véritable(s) chef(s)-d'œuvre»" ${ }^{14}$, et qui émaille tout au long le récit, et ce dès le chapitre $\mathrm{V}$, où la supériorité de la condition d'homme de guerre sur celle du courtisan est exposée à travers deux discours qui n'occupent pas moins de quinze pages.

8 Mais le consensus se fera plus sûrement sur l'attrait et l'originalité du récit de Jean de Bueil si l'on s'en tient au mode de représentation de certains épisodes et à l'instance narrative. En effet Le Jouvencel présente, surtout dans sa première partie, quelques descriptions qui tranchent agréablement sur celles stéréotypées et codées, de mise dans le roman médiéval. Ainsi à travers le tableau que dresse le narrateur d'une région dévastée par la guerre, puis des conditions précaires des hommes d'armes qui hantent cette région, l'insignifiant, le dérisoire, l'ordinaire accèdent à la littérature ${ }^{15}$. Peu de textes de cette époque laissent aussi bien percevoir au lecteur l'inconfort qui régnait dans des forteresses délabrées où le guetteur "n'estoit pas bien garanti contre le vent ", sans avoir rien à envier au sort du portier qui « estoit fort subject au chault et au hasle l'esté et l'yver au froit et à la gellée» ${ }^{16}$. Quant aux hommes et chevaux de la garnison au "chastel de Luc» c'est à qui sera le plus éclopé:

Et, pour brief parler, tant d'hommes que de chevaulx, les plusieurs estoient, les uns borgnes et les autres boiteux d'aucun membre, et n'y avoit cellui qui ne portast les enseignes de son mestier ${ }^{17}$

Et les premières prises du Jouvencel lorsqu'il s'attaque à l'ennemi, c'est-à-dire à la garnison de la forteresse voisine de Verset, n'ont rien de très glorieux: des chèvres, le linge d'une lessive «dont il fit son jacque ${ }^{18}$, puis la vache du capitaine de Verset. La pauvreté règne aussi à Verset, comme en témoigne la réaction du capitaine, «moult doullant» de la perte de la dite vache, «car sa femme en avoit du lait pour soy nourrir et son enfant» ${ }^{19}$, ou encore l'état des chevaux de la garnison "povres, mesgres et farcineux $»^{20}$, dignes répliques de ceux de Luc qui «jeunoient... le plus du temps; par quoy ilz estoient vains, chetifz et mesgres $»^{21}$. Et quand le héros passe de l'extrême pénurie à un premier équipement complet d'homme d'armes, avec son «jacque», son cheval « qui avait une grosse jambe derrière» et la « cuyrasse» de rechange du capitaine de Luc, son équipage n'est guère plus reluisant que celui dont Cervantes pourvoira don Quichotte. Il n'y a bien entendu aucune commune mesure entre les deux héros, car le Jouvencel est en tous points conforme à l'idéal chevaleresque, mais cette rencontre 
souligne la perspective de Jean de Bueil lorsqu'il développe le motif de la pauvreté de son personnage et de son entourage au début du récit. Pas de compassion, ni de commisération, mais le regard amusé de celui qui en est passé par là sur les privations et frustrations qui accompagnent la carrière des armes et le souci de montrer qu'elles trempent l'âme. A plusieurs reprises l'auteur du Jouvencel revient sur la dureté des conditions de vie des hommes d'armes. Déjà quand il commente les conditions de vie misérable des hommes de la garnison de Luc:

La grant povreté que c'estoit de leur fait, seroit bien difficile à racompter, ainsi qu'il est communement de toutes gens de guerre; car ilz sont nez et ordonnés à paine et travail; mais leur resconfort est du tout en Dieu, et aussi le hault voulloir et grant couraige qu'ilz ont et desir d'honneur avoir, et la louenge du monde qu'ilz acquièrent avecques le grant plaisir qu'ilz prennent à veoir et à aprendre de jour en jour choses nouvelles, les font joyeusement passer leurs souffraittes, dangiers, povretez et disettes qu'ilz ont à cause de la guerre...22

Ou encore quand il passe à la pauvreté de la forteresse adverse, celle de Verset. Le narrateur se demande au passage et non sans humour, ce que pouvaient bien chercher à gagner sur l'ennemi ces troupes ennemies, compte tenu de l'état de dénuement où elles se trouvaient, «car c'estoit toute povreté et n'avoient argent ne vivres, sinon très peu», puis il ajoute qu'il y avait pourtant parmi ces hommes " aucuns gentilzhommes bien nobles et de bon lieu» ${ }^{23}$, jeunes gens «là mys et ordonnés pour apprendre le mestier de la guerre et pour eulx endurcir au travail $»^{24}$. Suit alors un développement sur la nécessité dans l'apprentissage d'un métier de «fuyr les aises du corps, comme trop boire, trop mengier et trop dormir, et plusieurs autres delices charneulx», principe bien appliqué dans la formation des «estudians es ars liberaulx et en phillosophie moralle ou naturelle» ${ }^{25}$. Après avoir ainsi donné ses lettres de noblesse intellectuelle au métier des armes, Jean de Bueil revient aux "gens de guerre», et dénonce les effets pernicieux de l'abondance et du confort sur la «prouesse» de ces hommes qui sombrent «en lascheté et en paresse» ${ }^{26}$. Déjà les soldats d'Hannibal à Capoue... mais le récit, qui n'est pourtant pas avare de références à l'histoire antique notamment, n'en dit rien ici.

11 Ces développements sur la pauvreté en ouverture du récit jouent bien sûr un rôle dans la structure tripartite et ascendante du Jouvencel, qui fait passer le héros d'un état de quasi indigence à une situation moyenne puis fort élevée, et Jean de Bueil insiste sur ce mode de composition qu'il annonce en se référant à Aristote:

Si ay proposé... de deviser mon livre en trois principalles parties selon les trois manières de vivre recitées par le phyllosophe moral, qui sont: monostique, yconomique et pollitique. ${ }^{27}$ Mais c'est là leur moindre attrait. Ce qui attache bien davantage, on l'a déjà dit, c'est le mode ludique que choisit le narrateur pour représenter la misère de ses personnages.

Et plus largement ce narrateur offre au lecteur une situation narrative assez nouvelle puisque l'on se trouve dans «un récit à la première personne» où celui qui raconte l'histoire est non pas le héros mais le témoin des faits rapportés. Ce type de narration se rencontre à l'occasion dans les chroniques, et l'on peut penser à Froissart qui, chaque fois qu'il le peut-à partir du livre III des Chroniques- met l'accent sur sa présence auprès de grands personnages et par là sur la valeur de son témoignage. Cette situation d'un narrateur témoin de l'histoire d'un autre n'est pas inédite dans la fiction: on la rencontre dans le Jugement dou Roy de Behaingne ou dans La Fonteinne Amoureuse de Guillaume de Machaut, dans Le Dit dou Bleu Chevalier de Froissart, dans La Belle Dame sans mercy d'Alain Chartier et l'on pourrait multiplier les références ${ }^{28}$. Toutefois dans ces 
œuvres, qui ont toutes plus ou moins partie liée avec le lyrisme, elle n'intervient que ponctuellement dans le récit. De plus, comme Jacqueline Cerquiglini l'a bien mis en évidence, le témoin est un clerc qui fixe par l'écriture ce qu'il a entendu ${ }^{29}$ par hasardlamentations ou conversation d'un prince, d'un chevalier- et cette mise en scène engage toute une conception hiérarchique de la société - l'éternel débat du clerc et du chevalier $^{30}$.

Rien de tel avec Le Jouvencel: le narrateur se place d'emblée du côté des armes- «... des ma jeunesse, j'ay sieuvy les armes et fréquenté les guerres du très crestien roy de France, mon souverain seigneur, en soustenant sa querelle de tout mon petit povoir ${ }^{31}$. Et l'on peut penser avec Aragon que Jean de Bueil innove en donnant pour narrateur à son récit un personnage dont le rôle est celui d'un observateur et d'un enquêteur:

Le roman commence par un article qui a si souvent servi aux romanciers depuis Louis XI qu'il apparaît une pauvre chose aujourd'hui, mais on voudra se souvenir que c'était alors une pure invention: l'auteur d'abord parle à la première personne, dans le prologue et dans le premier chapitre, prenant soin de nous dire qu'il commence son livre à la vigile de l'Annonciation... et comment il arrive au château de Luc, dont il décrit encore en témoin, au chapitre II, la misère, et y fait paraître un jeune gentilhomme, qui sera le Jouvencel, auquel à partir d'ici est laissé entièrement la parole, bien qu'à la troisième personne, tout le restant du livre étant supposé noté par le Sire de Bueil sur les propos que le Jouvencel lui a tenus... ${ }^{32}$

Mais, si novatrice que puisse être l'instance narrative dans Le Jouvencel, il est sûr que ce choix n'est pas en priorité dicté par des préoccupations d'esthétique littéraire. Il découle de la visée première de l'œuvre qui, par le biais de la fiction, veut dispenser un enseignement pratique et moral sur l'art de mener la guerre... un Télémaque en quelque sorte pour hommes d'armes. Cet objectif est clairement exposé dès le prologue:

Si ay proposé à l'aide de Dieu escripre et compiller ung petit traictié narratif, pour donner cueur et voullenté à tous hommes, especiallement à ceulx qui sieuvent les adventures merveilleuses de la guerre, de tousjours bien faire et acroistre leur honneur et hardement de mieulx en mieulx. Et pour ce que, des ma jeunesse, j'ay sieuvy les armes et fréquenté les guerres... j'ay peu veoir par l'espace de long temps plusieurs et diverses manières de faire que les jeunes et nouveaux venus ne puent pas sçavoir de prime face. ${ }^{33}$

16 Transmettre des valeurs, mais aussi transmettre une expérience, et ce exclusivement à propos de la guerre, tel est bien le dessein déclaré du Jouvencel. Dès l'ouverture la guerre est au cœur du débat, introduite par une phrase inaugurale à la fois prolongement et reprise du début de la Genèse:

Au commencement de ce monde, après ce que Dieu eut creé l'homme et la femme et qu'il eut produit toutes choses pour servir et nourrir icellui, ne fut pas longuement la terre en paix. Ainçois commença la guerre.... ${ }^{34}$

17 D'entrée de jeu Jean de Bueil s'attache à montrer que la guerre est quasiment aussi vieille que le monde, puisqu'elle remonte à Caïn et Abel. Cela certes ne suffit pas à en faire un bien, mais c'est déjà justifier son existence par le droit que donne l'ancienneté. Honoré Bouvet avait du reste proposé mieux trois quarts de siècle auparavant dans son Arbre des batailles, puisque, à l'en croire, la guerre, ou plus exactement la première bataille, préexistait à la création du monde:

Si vous declaire que ce fut au ciel quant nostre Seigneur Dieu crea les angeles. Il en fit ung tant bel, tant noble et tant glorieux que de beauté il surmontoit toutes les creatures celestiales. Et tant resplendissoit sa clarté que toute la beauté des aultres mettoit au bas ainsi comme fait le grand cierge ardant qui abaisse la lumière d'une petite chandelle. ${ }^{35}$ 
18 On connaît la suite: grisé par sa resplendissante beauté cet ange voulut égaler Dieu. Alors commença la bataille entre les anges qui prirent son parti et «les bons angeles qui nullement ne vouloient soutenir celle opinion». D'où l'on peut déduire qu'il n'est pas étonnant qu'en ce bas monde surviennent guerres et batailles, "puisque elles furent premierement au ciel.» ${ }^{36}$

19 Faire remonter la guerre à la nuit des temps ne suffit cependant pas à lui assurer une garantie morale. Et Jean de Bueil en souligne le caractère maléfique quand il commente son origine: elle est née "à l'occasion d'envie», ou encore quand il la nomme «mortelle pestillence ${ }^{37}$. D'autre part, si la guerre s'impose par son ancienneté, elle s'impose aussi par son universalité:

Et pour ce que discord et discension de tout temps ont eu cours es parties du monde, ce n'est point à douter que, s'ilz ont cessé es aucunes parties de la terre, que ilz n'ayent tant plus renfforcé et renouvellé es autres. ${ }^{38}$

Et cette universalité permet d'introduire «les bons» à côté des «mauvais», de distinguer dans les raisons qui conduisent à faire la guerre:

combien que cellui n'est pas bon ne bien conseillé qui donne occasion de la (=la guerre ) commencer, neantmoins cellui n'est pas à reprouver qui la continue pour son droit garder et deffendre ${ }^{39}$

21 C'est du reste à partir de là qu'est né "le très noble et très excellent estat de chevallerie " afin de "conserver, deffendre et garder le pueple en tranquillité, qui communement est le plus grevé par les adversitez de la guerre $»^{40}$. L'exercice des armes peut donc, à certaines conditions, aller dans le sens de la morale chrétienne, il suffit pour cela qu'il s'attache à soutenir une juste cause- défense du droit, protection des faibles.

22 Le chemin avait été frayé depuis longtemps par les penseurs chrétiens qui, dès la conversion de l'empire romain au christianisme, avaient tenté de justifier la nécessité dans certains cas de recourir à la force pour le pouvoir temporel, comportement a priori peu compatible avec une religion basée sur l'amour du prochain et par là même respectueuse de sa vie ${ }^{41}$. Philippe Contamine souligne que la pensée de saint Augustin a joué sur ce point un rôle fondamental et largement influencé la pensée médiévale. Or l'évêque d'Hippone considère, dans La Cité de Dieu, que certaines guerres sont justes, quand elles sont menées pour défendre la paix ou soutenir la justice ${ }^{42}$. Cette notion de guerre juste on la retrouve ici, dans Le Jouvencel, à travers bien sûr de multiples intermédiaires. Parmi eux, peut-être le Livre des Fais d'armes et de Chevalerie, dont on sait qu'il était bien connu de Jean de Bueil ${ }^{43}$ Ou encore Honoré Bouvet, que Christine de Pizan reconnaissait comme l'un de ses maîtres, et qui présente, dans le premier chapitre de la quatrième partie de son Arbre de Batailles, une justification radicale de «bataille», et donc plus largement de la guerre, déclarant que:

... il est vérité que bataille n'est une male chose ainçois est bonne et vertueuse, car bataille ne regarde autre chose selon sa droite nature que retourner tort à droit et faire retourner dissension à paix selon le contenu de l'Escripture. ${ }^{44}$

Mais Jean de Bueil ne s'attarde guère sur la justification de la guerre. Une fois qu'il a établi que le métier des armes est compatible avec la volonté de Dieu, avec la morale chrétienne, il s'intéresse bien davantage sur le plan éthique et pratique au comportement de ceux qui y consacrent leur existence. ${ }^{45}$

En ce qui concerne la pratique de la guerre, Le Jouvencel entrelace habilement le fruit d'une expérience personnelle aux leçons tirées du passé, lointain-de fréquentes 
références aux épisodes guerriers de la Bible, aux historiens latins émaillent le texte ou proche-par exemple dans le discours du seigneur de Chamblay, les conseils qu'il donne pour s'assurer une victoire à partir des batailles de Shrewbury, Azincourt, Crevant, Verneuil, Baugé, Patay, Fremigny, Castillon et Auray ${ }^{46}$. Et bien sûr Jean de Bueil n'omet pas de faire intervenir Végèce, la référence par excellence en matière d'art militaire au Moyen Âge ${ }^{47}$.

Je laisse aux spécialistes de la pratique de la guerre le soin d'établir si Le Jouvencel apporte des éléments novateurs en ce qui concerne le métier des armes. Mais il est certain qu'il innove en matière de traité de guerre par le choix d'un exposé qui se détourne de la théorie, de l'abstraction et illustre par des cas concrets, dans le cadre de la fiction ou de l'histoire, ses idées sur l'efficacité en matière de combat. Car c'est bien d'efficacité qu'il s'agit et non de beaux coups d'épée et coups d'éclat. Un motif récurrent est celui de la surprise de l'ennemi, aussi les expéditions nocturnes abondent-elles et le souci d'effacer toute trace, d'étouffer tout bruit qui pourraient alarmer l'ennemi. Et il y a peu de place pour la prestance, l'élégance ${ }^{48}$, l'auteur n'hésitant pas à présenter ses personnages embusqués dans le fumier ou «le ventre contre terre... leurs sallades couvertes $»^{49}$ afin de s'assurer la victoire.

Mais l'auteur du Jouvencel ne recourt pas à la fiction seulement pour dispenser des conseils dans l'art de mener la guerre, il en use également pour communiquer son enthousiasme à l'égard du métier des armes. Car c'est très souvent à travers les propos de ses personnages que se dessine une image exaltée de la guerre, source de plaisir, moyen de promotion sociale, école d'ascétisme et instrument du salut éternel.

Et aussi à la vérité la guerre est une friande chose pour ung jeune homme comme vous, quant il y a le cœur. Mais qu'il ait tousjours Dieu et son honneur devant les yeulx et qu'il ne s'entremette fors de soustenir bonne querelle ! Car je croy que tout homme qui expose son corps à soutenir bonne querelle et à secourir son souverain seigneur ou son prouchain en bonne justice et en bon droit, fait et accomplist le commandement de Dieu. ${ }^{50}$

C'est en ces termes que le capitaine de Luc, qui est le premier à encourager la vocation du Jouvencel, présente la carrière des armes. La leçon ne sera pas oubliée par son protégé qui, devenu à son tour meneur d'hommes, déclarera après une opération réussie contre la ville d'Escallon:

C'est joyeuse chose que la guerre; on y oït, on y voit beaucoup de bonnes choses, et y apprent moult de bien. Quant elle est en bonne querelle, c'est justice, c'est deffendre droicture. Et croy que Dieu ayme bien ceulx qui exposent leur corps à vouloir faire la guerre et faire la raison aux ingratz et descongneuz, aux prosternés et orgueilleux, et qui vont contre bonne equité... c'est un plaisant mestier et bon à jeunes gens. Car ilz en sont amez de Dieu et du monde. ${ }^{51}$

Bien plus, le Jouvencel laisse entrevoir que la carrière des armes conduit au salut éternel aussi sûrement que la vie monastique:

... et, si Dieu plaist, nous acquerrons nostre sauvement à l'exercite des armes aussi

bien comme nous ferions à estre en contemplacion et à ne mangier que racines. ${ }^{52}$

La sobriété, du reste, n'est pas l'apanage des hommes de religion. Dans ce même discours, le héros rappelle à ses compagnons que ceux à qui Dieu a fait la grâce de leur donner un corps solide doivent l'exposer «pour la foy de Nostre-Seigneur Jhesus-Christ et pour soustenir bonne querelle» et éviter de «boire d'autant et faire choses dissollues et desraisonnables ne excès sans cause ${ }^{53}$ De plus dès le début de son ouvrage, on l'a vu, Jean de Bueil met l'accent sur les «souffraites, dangiers, povretez et 
disettes "qu'entraîne le métier des armes et juge nécessaire que les jeunes gens qui ont opté pour cette carrière se forment dans des conditions matérielles difficiles. Enfin, sur le mode comique, il présente a contrario les méfaits sur l'homme d'armes de la bonne nourriture et de l'inaction, quand le chancelier du roi invite son souverain à convoquer au plus vite ses troupes désœuvrées:

car ilz engressent trop; s'ilz sejournent plus, ilz ne pourront plus entrer en leur harnoix; et fauldroit que vous leur en donnassez de tous neufz..$^{54}$

Geoffroi de Charny avait déjà, dans son Livre de Chevalerie, plus d'un siècle auparavant, mis l'accent sur la nécessité pour le chevalier qui aspire à l'excellence de se détacher des plaisirs de la bonne chère et il recommandait la frugalité pour les jeunes gens ${ }^{55}$. Il avait également, et beaucoup plus nettement que Jean de Bueil, établi une comparaison entre les «saintes ordres de religion» et l'«ordre de chevalerie». Pour lui les frustrations et privations qu'imposait la vie religieuse se retrouvaient entières, voire amplifiées dans la vie du chevalier:

Et trop bien peut apparoir que es ordres de religion, combien qu'il leur soit dit à l'entrer, quant l'en cuidera mengier, l'en jeunera, quant l'on voudra jeuner, lors couvendra mengier, et quant l'en cuidera dormir, il convendra veillier, et moult de teles autres choses, n'est ce mie comparasons d'assez souffrir comme en l'ordre de chevalerie. Que qui voudroit considerer les paines, travaux, douleurs, mesaises, grans paours, perilz, froisseures et bleceures que li bon chevalier, qui l'ordre de chevalerie maintiennent ainsi comme il doivent, ont à souffrir et sueffrent mainte foiz, il n'est nulle religion ou l'en en sueffre tant comme font cil bon chevalier qui les faiz d'armes vont querant justement... ${ }^{56}$

31 Geoffroi de Charny se livrait alors à un passage en revue des mortifications corporelles, des dangers auxquels étaient confrontés les «bons chevaliers» et réaffirmait ce qu'il avait déjà avancé plusieurs fois: «certes en ceste ordre de chevalerie peut l'on tres bien les aumes sauver» ou encore: «Et qui fait les faiz d'armes plus pour avoir la grace de Dieu et pour les ames sauver que pour la gloire de ce monde, les ames dignes sont mises en paradis et sanz fin...»

Ces rencontres entre Le Jouvencel et Le Livre de Chevalerie n'ont rien de fortuit et la conviction que la pratique de la guerre, les hauts faits d'armes peuvent ouvrir les portes du paradis, si l'on combat pour une juste cause, est bien dans l'air du temps ${ }^{57}$. Mais il y a peut-être un rapport plus immédiat entre les deux textes et R. W. Kaeuper ${ }^{58}$ ainsi que $\mathrm{M}$. Vale ${ }^{59}$ pensent que Jean de Bueil connaissait l'œuvre de Geoffroi de Charny, ce qui conforte bien une lecture du Jouvencel comme un traité de guerre qui aborde, par le biais de la fiction, outre les aspects ci-dessus envisagés, une réflexion politique, juridique et morale.

33 Ce n'est pourtant pas à ce type de réception que nous convie Guillaume Tringant quand il rédige son «exposition du livre du Jouvencel» ${ }^{60}$. Cet écuyer de Jean de Bueil se livre en effet à des révélations qui font de l'ouvrage de son seigneur une autobiographie déguisée et qui, de plus, si l'on démêle sans erreur l'expression et la construction des plus embrouillées du début de ce commentaire, font de cette œuvre le fruit de la collaboration de familiers de Jean de Bueil, d'une équipe de nègres avant la lettre.

La raison d'être de ce «déguisement» serait la modestie du maître de Tringant, argument dont usait déjà Christine de Pizan quand dans son Livre des Fais d'armes et de Chevalerie elle déclarait actualiser l'enseignement de Végèce et autres autorités par le "conseil des saiges chevaliers expars esdictes choses d'armes», qui par modestie 
refusaient d'être nommés. ${ }^{61} \mathrm{Et}$ cette modestie n'a pas été sans poser problème aux rédacteurs du Jouvencel, comme le souligne l'auteur du commentaire:

Et pour ce que mes maistres... ne voloient pas declarer les noms ne les lieux où les chouses ont esté faictes, ou de ceulx qui les ont faictes, il leur a convenu querir noms estranges, qui les a faict troubles; car, quant de tous points ilz eussent escript les noms et les lieux, ilz l'eussent faict plus legièrement et myeux à leur plaisir; mais le sire de Bueil, à qui eulx et moy estions, ne vouloit jamaiz qu'ilz le fissent pour ce qu'il ne voloit estre loué ne magnifié devant luy-mesme; car la plupart des chouses qui sont escriptes oudit livre du Jouvencel, il les a faictes et executées... ${ }^{62}$

Guillaume Tringant revient un peu plus loin sur cette volonté délibérée du seigneur de Bueil de brouiller les cartes, dictée par le souci de ne pas se mettre en avant et céder à un vain orgueil et il précise:

Les choses mises à pars soy eussent esté trop cleres, et mondit maistre ne le voloit pas; car il ne donnoit point d'argent pour soy faire mettre ès Croniques. ${ }^{63}$

Camille Favre et Léon Lecestre ont pris très au sérieux les révélations de Tringant et complètent chaque fois qu'ils le peuvent les références de l'auteur du commentaire ou avouent leur impuissance, quand ils n'ont pu identifier une ville, un événement. Par ailleurs Camille Favre dans son introduction littéraire fait intervenir d'autres raisons qui auraient contribué à faire du Jouvencel une sorte de roman à clés. Ainsi la crainte de nuire au succès du roman en lui donnant une actualité passagère et en accentuant le caractère historique aux dépens de l'intérêt romanesque et didactique ${ }^{64}$. Ou encore des raisons d'ordre politique, Jean de Bueil cherchant à éviter de se mettre trop en évidence dans une œuvre écrite sous le règne de Louis XI, mais qui se livre à un éloge en règle du règne précédent, celui de Charles VII.

Aragon ne croit guère à cette modestie, pas plus qu'à cette prudence et son argumentation est tout à fait convaincante. Quant à G. W. Coopland, il considère pour sa part que si Le Jouvencel est l'une des plus intéressantes productions littéraires de la fin du Moyen Âge, ce n'est certes pas en tant que roman à clés, car dans cette perpective il est clumsy and inconsistent ${ }^{65}$. Et la prestation de Guillaume de Tringant n'est pas non plus portée aux nues par J. Blanchard quand il juge que l'écuyer de Jean de Bueil «n'a pas été sensible aux raisons d'être de la compilation (c'est-à-dire Le Jouvencel ) et a transformé le livre en récit d'une vie, en une chronique des faits et gestes.»" ${ }^{66}$

Cette transformation ne s'opère pas sans mal, comme en témoignent certaines besogneuses explications du commentaire ${ }^{67}$. Et surtout revient avec insistance le souci des rédacteurs du Jouvencel de masquer les faits et personnages réels. Recours à des noms de gens et de lieu inconnus "pour mieulx deguiser le livre», «chouses meslez afin que l'on n'en eust point de cognoissance et qu'on n'y peust ryens gloser qui c'estoit ne dont ce venoit ${ }^{68} \mathrm{Ou}$ encore l'utilisation d'un seul nom renvoyant à plusieurs lieux ou personnages. Crator, nous dit l'«exposition» renvoie à Orléans, Lagny et Sablé, superposition concertée car «les choses mises à pars soy eussent esté trop cleres, et mondit maistre ne le voloit pas ${ }^{69}$. Et derrière le comte de Parvencheres se cachent plusieurs seigneurs dont Jean de Bueil a été le lieutenant, mais «pour mieulx deguiser tousjours icelluy livre on les appelle tous le conte deParvancheres.» ${ }^{70}$ On pourrait encore relever le passage où, tâchant de démêler personnages fictifs et personnages réels dans Le Jouvencel, Tringant ajoute:

car on faisoit sur le livre ce qu'on povoit pour n'y entendre ryens. Ainsi estoient les chouses meslées l'une parmy l'autre; mais touteffoys c'estoient chouses advenues et 
y eust eu quelque entendement, ce que ne voloient ceulx qui faisoient le livre dudit Jouvencel.

Qu'a donc cherché à faire Guillaume de Tringant en passant outre à la volonté de ses «maistres»? Car l'essentiel de sa démarche correspond à une entreprise de dévoilement, qui n'a de raison d'exister qu'à partir d'un dessein concerté de dissimulation. Sans doute, à en croire ses déclarations, il a rompu ce pacte du silence afin de rendre au seigneur de Bueil sa part de gloire- car «la plupart des chouses qui sont escriptes oudit livre du Jouvencel, il [= Jean de Bueil] les a faictes et executées, dont d'autres se sont donné la gloire de les avoir faictes $»^{71}$. Tringant se serait donc lancé dans une sorte de biographie chevaleresque afin d'arracher à l'oubli les hauts faits de son seigneur et afin de lui rendre- malgré lui !- justice.

Toutefois, à y regarder de plus près, on remarque que les élucidations de l'écuyer n'occupent pas, loin de là, tout le champ de son intervention. Le récit des événements militaires dont il a été informé, et auxquels a participé- mais pas toujours !- Jean de Bueil, vient souvent occuper le devant de la scène. Tringant lui-même en a conscience, et, après avoir évoqué la bataille de Verneuil, puis relaté une anecdote concernant un Anglais qui pour tirer une meilleure rançon de son prisonnier l'avait pendu par les pieds, puis encore fait mention du désastre d'Azincourt, il enchaîne:

$\mathrm{Et}$, pour retourner à ma matière et à parler du Jouvencel... ${ }^{72}$

41 On peut donc se demander si l'auteur de cette "exposition» n'a pas eu la tentation de faire à sa manière, en fonction de ce qu'il avait entendu, des témoignages qu'il avait pu recueillir, œuvre de chroniqueur. Démarche quelque peu naïve qui consisterait à se découper un petit canton d'immortalité en s'attachant à une œuvre dont le succès fut considérable dès sa diffusion.

Quoi qu'il en soit, si l'intervention de Guillaume de Tringant n'apporte rien au Jouvencel, elle ne lui enlève rien non plus et même contribue à faire apparaître, par le rapprochement entre éléments de la fiction et éléments de la réalité, «l'invention du roman ou réalité dominée» ${ }^{73}$. Et en fin de compte, si l'on devait décerner quelques lauriers à Guillaume de Tringant, ce serait dans le registre de la critique ou de l'analyse littéraire...

Il n'en demeure pas moins que «le propos essentiel» (Aragon) de Jean de Bueil est d'apporter sa contribution didactique et morale à la conception et à la pratique de la guerre. Mais ce qui sauve Le Jouvencel et l'empêche de prendre place parmi les «fastidieuses compilations $»^{74}$ relatives à l'art militaire de la fin du Moyen Âge, c'est la présence en arrière plan d'une expérience personnelle et l'éloge en règle qu'il propose de la guerre par l'intermédiaire de ses personnages. L'auteur s'inscrit par ce dernier trait dans la lignée des auteurs qui furent aussi des «seigneurs des batailles», pour qui la guerre «représentait tout, une profession comme un sport, un moyen de s'enrichir comme la justification de leurs privilèges juridiques et de leur prééminence sociale.» ${ }^{75}$ Lointain héritier de Bertrand de Born, Jean de Bueil doit peut-être à ses convictions sur la grandeur et la dignité de la carrière des armes d'avoir échappé au scepticisme face à «l'incertitude du monde " $^{76}$ de ceux que Jean Dufournet appelle «la génération de Louis XI», bien représentée par des œuvres aussi différentes que Le Testament de Villon, La farce de maistre Pathelin,Les Cent Nouvelles Nouvelles ou les Mémoires de Philippe de Commynes. Le héros du Jouvencel, lui, garde jusqu'au bout ses certitudes et l'un de ses derniers messages est que «l'on ne doit point revocquer en doubte que l'art de 
bataille ne soit la meilleure chose de toutes les autres choses. $\gg^{77}$ Art dont Voltaire dira deux siècles plus tard:

Cest sans doute un très bel art que celui qui désole les campagnes, détruit les

habitations et fait périr, année commune, quarante mille hommes sur cent mille. ${ }^{78}$

\section{NOTES}

1. Aragon, La mise à mort, Gallimard, Folio, rééd.1988, p.189.

2. Le Jouvencel, éd. C. Favre et L. Lecestre, Paris, S.H.F., 1887-1889, vol. I, p.15. Toutes les références du présent article renverront à cette édition. On pourra consulter sur cet ouvrage le bel article d'Elisabeth Gaucher, «Ecriture de soi, écriture du politique: le Jouvencel », à paraître dans les Mélanges Françoise Autrand.

3. Grundiss der Romanischen Literaturen des Mittelalters, vol. VIII, La littérature française aux XIVe et $X V^{e}$ siècles, sous la direction de D. Poirion, t. 1, Heidelberg, 1988, pp.354-358.

4. Op.cit., I, p.22.

5. Ibid., p.23.

6. Ibid.

7. E. Baumgartner, Le récit médiéval, Hachette, 1995, p.104.

8. Op.cit., II, p.266.

9. Ibid. p.209.

10. Ibid.

11. Ibid. p.210.

12. Ibid.

13. Ibid.

14. P.Paris, Les manuscrits françois de la bibliothèque du Roi, Paris, 1838, vol. II, p.139.

15. Cette accession, on la trouve déjà dans la nouvelle au XV $\mathrm{XV}^{\mathrm{e}}$ siècle, mais non, me semble-t-il, dans les romans, chroniques ou ouvrages didactiques.

16. Op.cit., I, p.20.

17. Ibid., p.22.

18. Ibid., p.24. Rappelons que le «jaque», comme le mentionne en note l'éditeur du Joucencel, désigne «un justaucorps de cuir, rembourré de vieux linge ou de bourre, à l'épreuve de l'épée». On admirera le sens de la récupération et du bricolage que développe chez le Jouvencel son extrême dénuement !
19. Ibid., p.25.
20. Ibid.
21. Ibid., p.22.
22. Ibid., p.21.
23. Ibid., p.25.

24. Ibid.

25. Ibid., p.26.

26. Ibid.

27. Ibid., p.16-17.

28. Voir J. Cerquiglini, «Un engin si soutil», Guillaume de Machaut et l'écriture au XIV siècle, pp. 113-114. 
29. Pour le dit de Froissart et La Belle Dame sans mercy, le témoignage porte également sur ce qui a été vu par le narrateur, mais cette relation ne fait que dramatiser les paroles entendues et rapportées.

30. Pour la très pertinente mise en rapport de la hiérarchie sociale et de la hiérarchie des sens on se référera aux pages 112-114 d' «Un engin si soutil», mais également à deux articles du même auteur: «Le clerc et le louche: Sociology of an esthetic», «Poetics Todays», V (1984), pp.479-491, et «L'écriture louche. La voie oblique chez les Grands Rhétoriqueurs», Les Grands Rhétoriqueurs, Actes $\mathrm{du} \mathrm{V}^{\mathrm{e}}$ Colloque International sur le Moyen Français. Milan, 6-8 mai 1985. Vol. I, Milan, 1985.

31. Op.cit., p.15.

32. Aragon, op.cit., pp.192-193.

33. Op.cit., p.15.

34. Ibid., p.13.

35. Honoré Bouvet, L'Arbre des batailles, éd. E. Nys, Bruxelles, 1883, p.4.

36. Ibid.

37. Op.cit., p.13.

38. Ibid., p.14.

39. Ibid.

40. Ibid.

41. Et déjà dans l'Ancien Testament parmi les commandements transmis par Yahvé à Moïse figure: «tu ne tueras pas» (Exode, 2013$)$.

42. Sur la réflexion concernant la guerre dans l'Antiquité tardive et au Moyen Âge, on pourra consulter Philippe Contamine, La guerre au Moyen Âge, PUF, $3^{\mathrm{e}}$ édition, 1992, pp.423-477.

43. Cf. G. Coopland, «Le Jouvencel revisited», Symposium, t. 5, 1951, pp.137-186. Ou encore M.H.Keen, «Chivalry, Nobility and Man at Arms», dans War, Literature and Politics in the late Middle Ages, Liverpool, 1976, p.36.

44. Op.cit., p.83.

45. Ce déplacement étant, selon Franco Cardini, un trait caractéristique de la «chevalerie». Cf. La culture de la guerre, Gallimard, 1992, p.323.

46. Op.cit., pp.61-65.

47. Sur la vogue de Végèce à la fin du Moyen Âge voir P.Contamine, G.R.L.M.A., VIII, pp.349-352.

48. On pourrait rapprocher cette mise à distance de l'esthétique de l'indifférence du héros de Jean de Bueil à l'égard de l'élégance vestimentaire: cf. I, p.101. Voir à ce propos l'article de C. Marchello-Nizia, «Codes vestimentaires et langage amoureux au XV siècle», Europe, $\mathrm{n}^{\circ} 654$, octobre 1983, pp.36-42.

49. Voir par exemple p.87, p.89, p.115. On notera l'effet de contraste dans l'opération de camouflage des «sallades» avec le topos souvent présent dans les chroniques du flamboiement des casques sous le soleil.

50. Op.cit., p.118.

51. Ibid., II, p.20.

52. Ibid., p.21.

53. Ibid.

54. Ibid., II, p.165.

55. R. W. Kaeuper and E. Kennedy, The Book of Chivalry of Geoffroi de Charny,Text, Context, and Translation, University of Pennsylvania Press, Philadelphia, 1996, p.110, pp.122-124.

56. Ibid., pp.174-176.

57. On la retrouve chez Honoré Bouvet et Christine de Pizan. Il paraît d'ailleurs évident que dans une société où la pensée religieuse est encore fondamentale, on ne peut exalter la carrière des armes qu'à condition de prendre en compte le salut éternel de celui qui opte pour elle.

58. The Book of Chivalry of Geoffroi de Charny, op.cit. 
59. War and Chivalry: Warfare and Aristocratic Culture in England, France and Burgundy at the End of the Middle Ages. Norwich, 1981.

60. Le Jouvencel, éd. cit., II, p.265.

61. P.Contamine, GR L M A, VIII, 1, p.355.

62. Op.cit., II, pp.266-267.

63. Ibid., II, p.283.

64. Ibid., I, p.CCCIX.

65. Symposium, t. 5, p.182.

66. J. Blanchard, «Ecrire la guerre au XV siècle», Le Moyen Français, 24-25, 1990, p.10.

67. Voir par exemple p.284 à propos du Jouvencel prisonnier ou p.293 à propos de «la fiction du roi Amydas».

68. Op.cit., II, p. 279.

69. Ibid., p.283.

70. Ibid., p.298.

71. Ibid., p.267. Ou encore, p.290: «Après ces chouses advenues et faictes par mondit maistre le Jouvencel (je les escriptz en gros, afin que chacun sache qui c'est qui les a faictes...)»

72. Ibid., p.269-270.

73. Aragon, La mise à mort, p.194.

74. P.Contamine, op.cit., p.354.

75. F. Cardini, La culture de la guerre, op.cit., p.413.

76. Jean Dufournet, Villon: ambiguité et carnaval, Champion, 1992, p.129.

77. Op.cit., II, p.258.

78. Dictionnaire philosophique, «Guerre», Garnier, 1967, p.228.

\section{AUTEUR}

\section{MARIE-THÉRÈSE DE MEDEIROS}

Université d'Orléans 